\title{
Correlation between cutaneous and ocular involvement during Lyell syndrome and Stevens-Johnson syndrome in children
}

\author{
Kenza Kandri Rody ${ }^{*}$, Mariam Zakoun ${ }^{2}$, Sarah Belghmaidi ${ }^{2}$, Ahmed Ghazza $^{2}$, Ibtissam Hajji ${ }^{2}$, Abdeljalil Moutaouakil ${ }^{2}$,
} Said Amal ${ }^{1}$, Ouafa Hocar ${ }^{1}$

${ }_{1}^{1}$ Department of Dermatology, CHU Mohamed VI Marrakech, Morocco
${ }^{2}$ Department of Ophthalmology, CHU Mohamed VI Marrakech, Morocco

DOI: $10.36347 /$ sasjm.2021.v07i03.006

| Received: 14.02.2021 | Accepted: 19.03.2021 | Published: 28.03.2021

*Corresponding author: Kandri Rody Kenza

Abstract

Original Research Article

Lyell syndrome and Stevens-Johnson syndrome (SJS) are two rare but potentially life-threatening mucocutaneous disease. The aime of our study is to describe their cutaneous, mucosal and ophthalmological damage in children, and to study the correlation between the severity of cutaneous and ocular damage based on the prognostic scores of the disease. It's a retrospective study involving 16 files, collected over a period of 4 years, and retained before the existence of an associated eye injury. The average age of children was 7 years with a 5/3 sex ratio. 9 of our patients were classified according to the SCORTEN score in score II, 4 in score IV and 3 in score V whose evolution was unfortunately death. Ocular damage has been reported in all our patients, it interested at the acute phase two sites: conjunctiva and cornea, and at late phase eyelid architecture abnormalities. All of our patients had dry eyes syndrome and one case of corneal blindness was reported. According to Foster's classification, 7 patients were classified as Stage I, 5 as Stage II and 4 as Stage III. Stage 1 included $86 \%$ of SJS, Stage II 22\% of SJS and $42.85 \%$ of Lyell, while Stage 3 included only Lyell cases, which make the correlation between the severity of ocular and cutaneous involvement and the prognosis of the disease. Lyell's syndrome and Stevens-Johnson syndrome are most often drug-induced dermatological emergencies. They can engage the vital prognosis in 1/3 of cases as well as the functional and aesthetic prognosis by their cutaneomucosal and ocular damage. There is a correlation between the severity of ocular and cutaneous involvement as well as the overall severity of the disease. This is quite evident while seeing the association of severe stages according to the Foster classification to the most severe stages of SCORTEN score.

Keywords: Lyell syndrome, Stevens Johnson syndrome, correlation, cutaneous involvement, ocular involvement.

Copyright $(\mathcal{C} 2021$ The Author(s): This is an open-access article distributed under the terms of the Creative Commons Attribution 4.0 International License (CC BY-NC 4.0) which permits unrestricted use, distribution, and reproduction in any medium for non-commercial use provided the original author and source are credited.

\section{INTRODUCTION}

Lyell syndrome and Stevens-Johnson syndrome (SJS) are serious dermatological emergencies most often drug-induced [1]. They are rare, but plagued by high morbidity and mortality. The lesions are mainly cutaneous-mucous and ocular [2]

The objective of our work is to describe the dermatological and ophthalmic complications in children in the course of Lyell syndrome and Stevens Johnson syndrome, and investigate the correlation between the severity of skin and eye damage based on prognostic disease scores [3].

\section{PATIENTS AND METHODS}

This is a retrospective study in collaboration between the dermatology and ophthalmology department, involving 16 cases of Stevens Johnson and Lyell, diagnosed according to the criteria of BastujiGarin et al. [4] and including only children under 15 years of age over a four-year period from May 2015 to May 2019. The severity of the disease and the extent of the lifted skin were assessed by the Scorten score, and the ocular involvement by the Foster score.

A report has been prepared for all patients including the following criteria: age, sex, medical history, drug intake, skin and mucous manifestations, data from daily ophthalmological examination, systemic manifestations, paraclinical examinations, duration of hospitalization, treatment and evolution.

Scorten's score for the items described in Table 1 was calculated. Its value varied from 1 to 7 by giving a point to each item [3]. Ophthalmological 
examination data allowed us to classify the ocular involvement into four stages according the Foster classification (Table 2) [5]. Drug imputability was determined using intrinsic and extrinsic imputability
[6]. A follow-up of patients was carried out after discharge according to the severity of the injury assessment. Cutaneous and ocular sequelae were noted during patient follow-up.

Table-1: SCORTEN parameters

\begin{tabular}{|c|c|}
\hline Paramètres & Points \\
\hline Age $\geq 40$ years & 1 \\
\hline Malignancy & 1 \\
\hline Initial surface of epidermal detachment $\geq 10 \%$ & 1 \\
\hline Tachycardia $\geq 120 / \mathrm{min}$ & 1 \\
\hline Serum urea $\geq 10 \mathrm{mmol} / 1$ & 1 \\
\hline Serum glucose $\geq 14 \mathrm{mmol} / 1$ & 1 \\
\hline Bicarbonate $<20 \mathrm{mmol} / 1$ & 1 \\
\hline
\end{tabular}

Table-2: Foster classification

\begin{tabular}{|c|l|}
\hline Stage & Clinical features \\
\hline I & Chronic conjunctivitis with subepithelial fibrosis \\
\hline II & Shortening of inferior fornix \\
\hline III & $\begin{array}{l}\text { Symblepharon /kertopathy / corneal neovascularisation/ trichiasis/distichiasis /conjonctival } \\
\text { keratinisation/ tear deficiency }\end{array}$ \\
\hline IV & Ankyloblepharon/Sever dry eye syndrome / xerosis \\
\hline
\end{tabular}

\section{RESULTS}

We identified 16 hospitalized patients with Lyell's syndrome or Stevens Johnson's syndrome during the four years of the study period. The average age of the children was 7 years with a sex ratio of $5 / 3$. The drug origin was incriminated in all our patients. The diagnosis was retained when finding bullous lesions and mucous erosions interesting $<10 \%$ of the skin surface in case of SJS ( 9 cases or $56.25 \%$ ) (Figure 1 ), and $>30 \%$ in case of Lyell syndrome ( 7 cases or $43.75 \%$ ) (Figure 2, 3), after taking medication with an average delay of 10 days. The drugs in question were dominated by beta-alactamines (amoxicillin: 32\%) and antiepileptics (sodium valproate: $22 \%$, tegretol: $16 \%$ ). 9 of our patients were classified according to the SCORTEN score in II (56.25\%), 4 in IV (25\%) and 3 in $\mathrm{V}(18.75 \%)$ (Figure 4). The skin condition interested the entire integument, especially the face, trunk and limbs. Mucosal involvement in all children was represented mainly by hemorrhagic erosions of the oral cavity, and by ocular involvement. The latter was bilateral and interested at the acute phase two sites: conjunctiva with hyperhemic conjunctivitis (100\%), and cornea: superficial spotted keratitis (80\%) (Figure 5), corneal ulcer (60\%) (Figure 6) and pseudo-membranous keratoconjunctivitis $(40 \%)$. In the late phase, ocular involvement consisted mainly of abnormalities of the palpebral architecture with fibrous inflammation of the eyelids (Figure 7): a symbleppharon (25\%), an entropion (12.5\%), a trichiasis (6.5\%). All of our patients had dry syndrome and blindness was reported. According to the Foster classification, 7 patients had mild impairment and were classified as Stage I (43.75), 5 moderate Stage II (31.25), 4 severe Stage III (25\%). Stage I included $86 \%$ of SJS, Stage II $22 \%$ of SJS and $42.85 \%$ of Lyell, while Stage III included only Lyell cases (Figure 8) correlating the severity of ocular involvement with skin involvement and the prognosis of the disease.

The therapeutic management was symptomatic and consisted initially of stopping the drug in question and the intensive care: oxygen therapy, hydroelectrolyte and nutritional intake, antibiotic therapy in case of signs of infection and thromboembolic prevention. For cutaneous damages, daily local care was carried out by creams based on fusidic acid or sulfamides and fatty tulles after piercing of the bubbles, until reepidermisation. Despite a relay with scar creams, the majority of patients kept diffuse dyschromic scar macules in the sequellary phase. Ophthalmologically, the initial management consisted of eye care with debridement of the conjunctival cul-de-sac associated with antibiotic and corticosteroid eye drops with artificial tears without preservatives. A symblepharon prevention ring has been implemented in all patients. After stabilizing the lesions, 4 patients benefited from a release of the symblepharon with reconstruction of the cul de sac by amniotic membrane, 2 patients benefited from a cure of entropion. A multilayer amniotic membrane graft was performed in 3 patients for chronic ulcer, with no improvement in a patient whose progression was marked by corneal opacification and neovascularization leading to corneal blindness (Figure 9).

The average duration of hospitalization was 35 days (20-57 days) and the evolution was marked by 3 deaths (classified as Stage $\mathrm{V}$ according to the SCORTEN score). 
Kenza Kandri Rody et al., SAS J Med, Mar, 2021; 7(3): 90-95

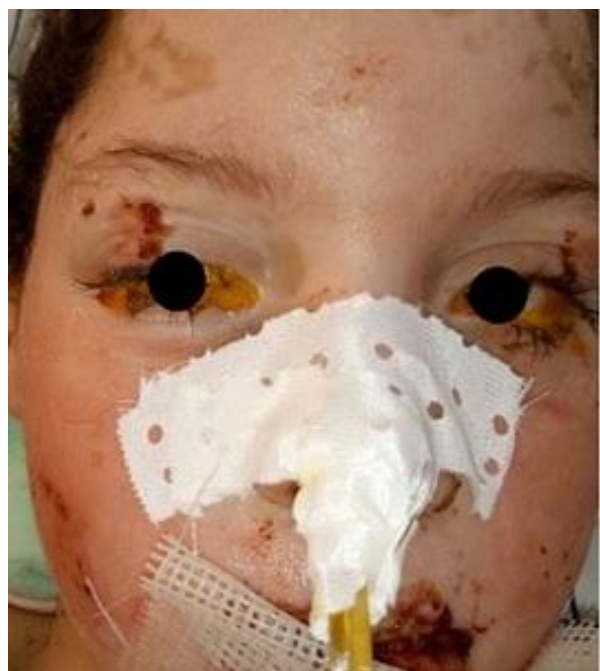

Fig-1: Stevens Johnson syndrome with ocular involvement and oral and nasal mucosal damage

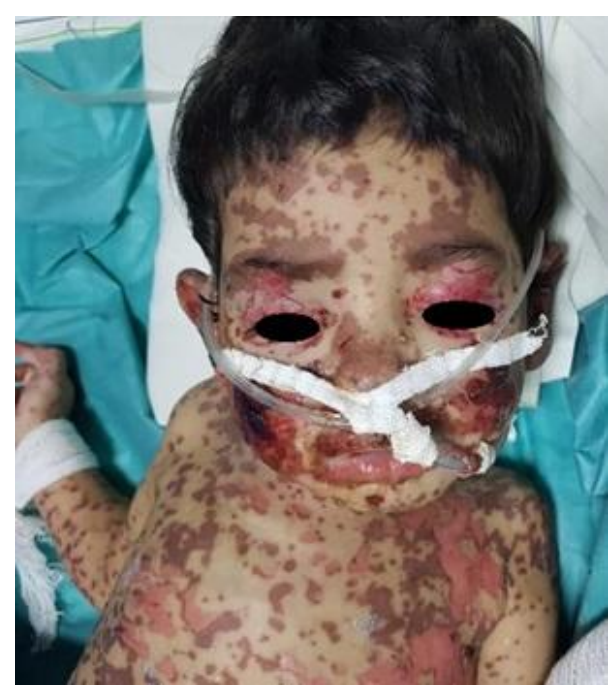

Fig-2: Cutaneous detachement with ocular and oral mucosal damage in a child with Lyell syndrome

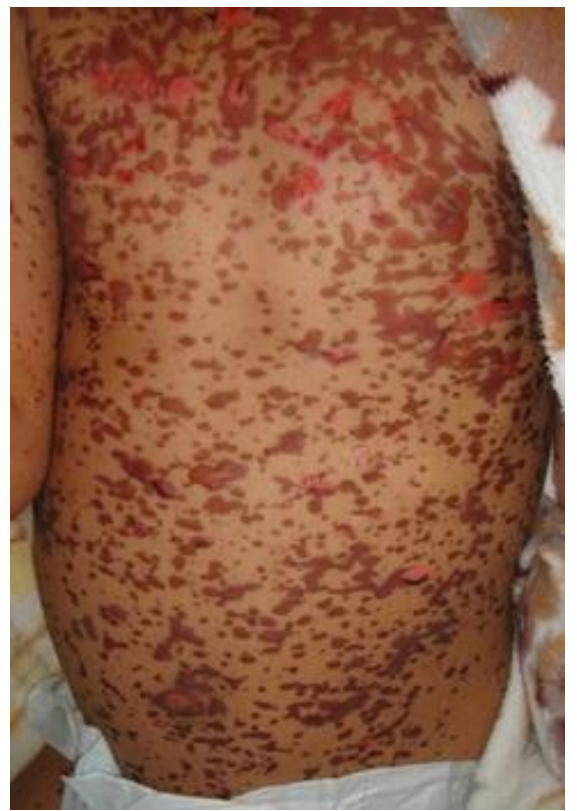

Fig-3: skin lesions during Lyell syndrome in a child

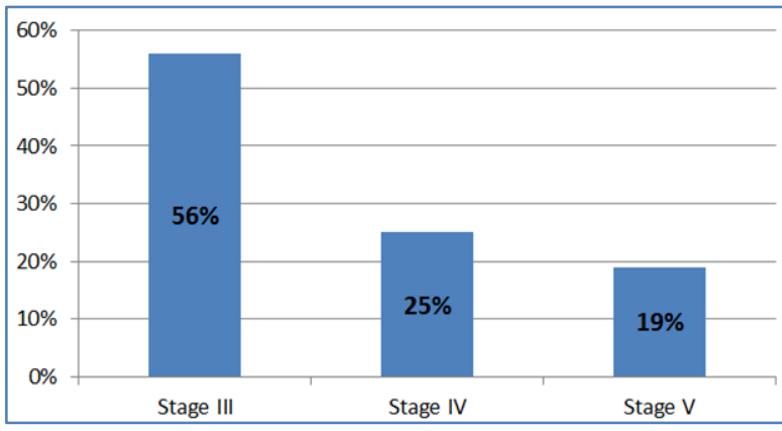

Fig-4: Distribution of patients according to Scorten's prognosis score

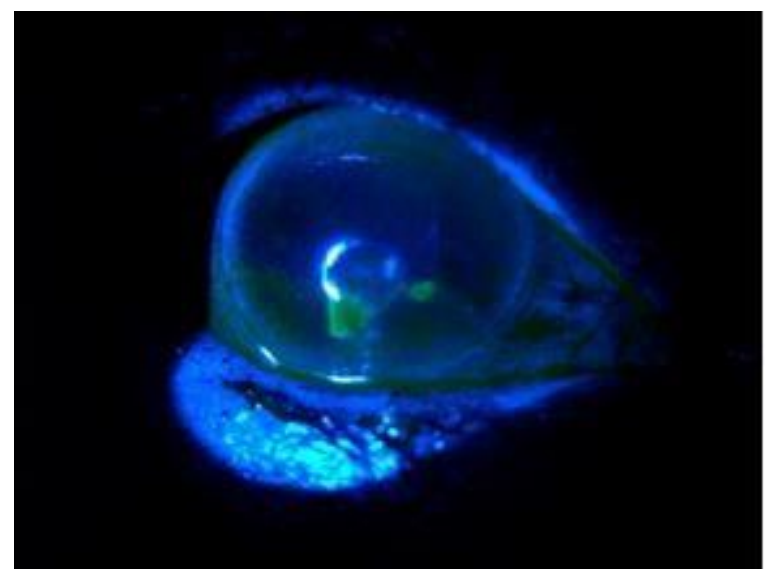

Fig-5: Superficial punctate keratitis with corneal ulcer in a patient with Stevens Johnson syndrome

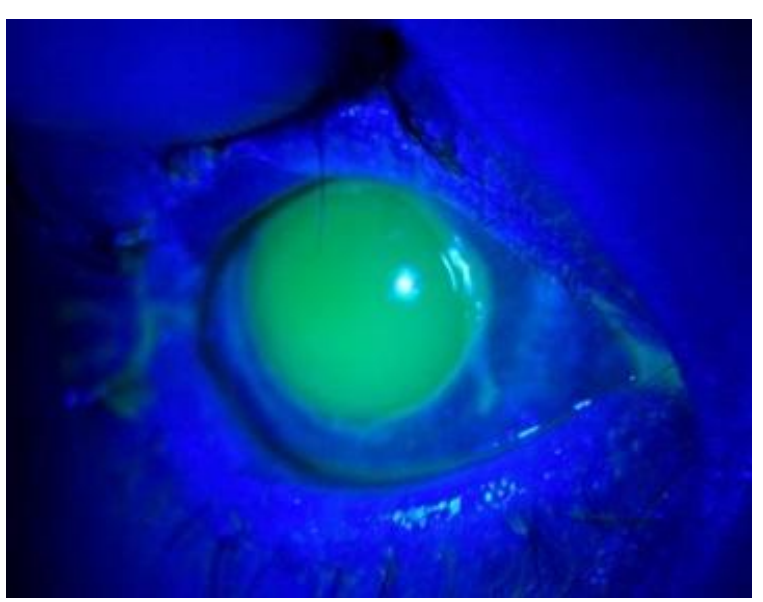

Fig-6: Total corneal ulcer

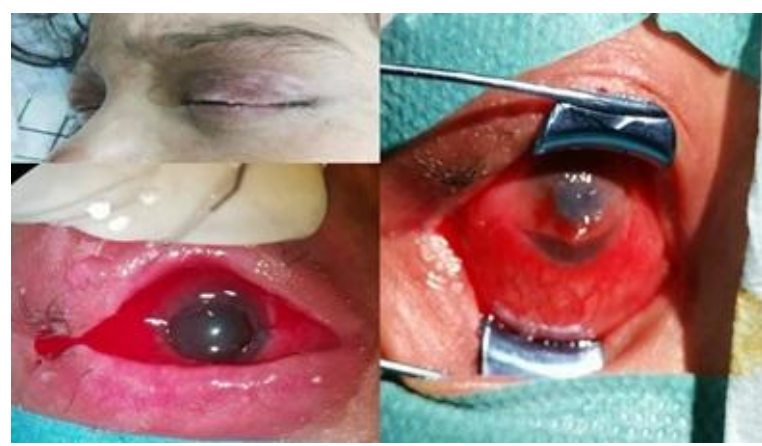

Fig-7: Sequellar stage of a Stevens Johnson syndrome with fibrosing inflammation of the eyelids and chronic bilateral corneal ulcer 


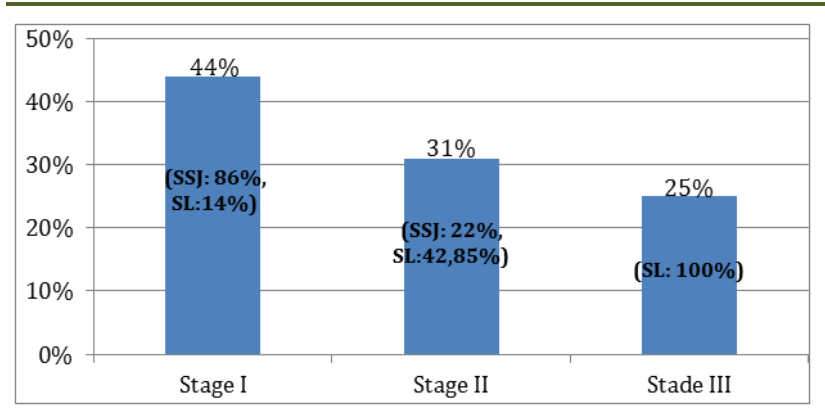

Fig-8: Ocular damage classification according to Foster's score

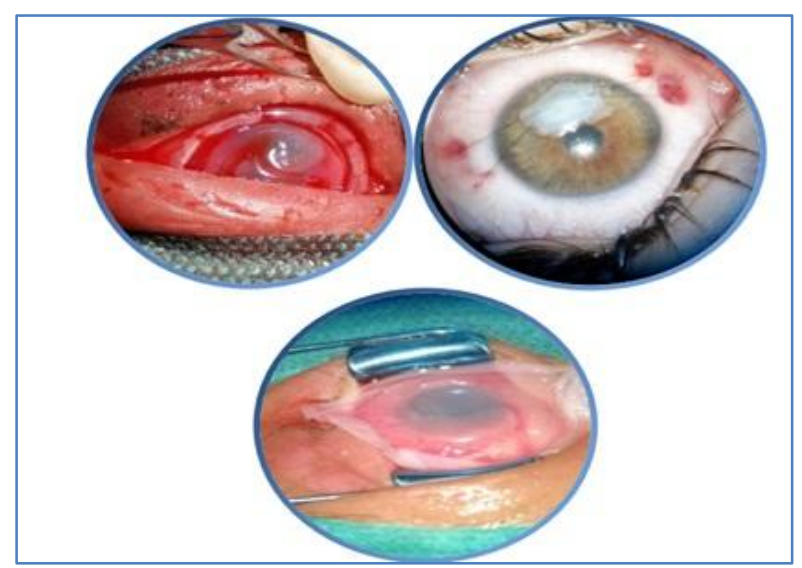

Fig-9: a) Placement of a symblepharon ring; b) Multilayer amniotic membrane transplant; c) Overlay amniotic membrane transplant

\section{DISCUSSION}

Lyell and Stevens Johnson syndromes are serious and rare toxidermis [7, 8]. The incidence of these conditions is estimated between 1 and 2 cases per million inhabitants per year [9]. They can affect all age groups with a clear predominance in polymedicated older populations. In our series, we focused only on children in order to explore the particularities of this age group. The literature reports a slight female predominance, with a sex ratio of 0.6 [10], unlike our study where the predominance was male. The risk factors identified in the literature are mainly: bone marrow transplant, and HIV infection. None of our patients had these risk factors. The distribution of the most incriminated drugs varies according to age, terrain, genetic and socio-economic characteristics of the different populations: predominance of betalactamines in our study joining the study of a Moroccan adult population of Mouafik et al. [11]. Unlike the predominant antiepileptics in Tunisia, antiinflammatory drugs in Japan [12], and sulfamides in Côte d'Ivoire [13]. Note that the occurrence of these syndromes is independent of the dose of medication received and minor exposure may trigger the disease.

Lyell and Stevens Johnson syndromes are considered varying degrees of severity of the same condition. They share common physiopathological, clinical and histological characteristics and are distinguished by the extent of the discoloured surface
[11]. Histologically, there is keratinocyte necrosis and alteration of the dermal junction [14]. Clinically, they are characterized by a predominant detachment at the face, neck and trunk and extending rapidly to the entire integument [15-17]. A decoupled skin surface greater than $30 \%$ argues in favour of Lyell syndrome, which clearly differentiates it from SJS (decoupled SC less than $10 \%$ ). Mucosal lesions are almost constant and can precede the skin signs. The alteration of the general state associated with visceral involvement (respiratory, renal, hematological, etc.) can complicate the clinical picture. The lesions usually begin 4 to 28 days after taking a drug (10 days in our study) and can continue to evolve for a few days, even after stopping the drug in question [11].

Among mucosal signs, ocular involvement is found in 85 to $95 \%$ of patients; it is mainly located at the level of the anterior segment and annexes. A large clinical polymorphism has been found in literature ranging from catarrhal conjunctivitis or superficial punctuated keratitis to severe forms that can lead to blindness. The most common long-term ocular complication found in the literature is dry syndrome [18], joining our study. Bilaterality of eye damage was found in all studies. The intensity of the acute stage of the disease determines the severity of the sequelae. These represent the main problem and make the severity of these conditions [19-25].

Lyell and Stevens Johnson syndromes require early and multidisciplinary management. The treatment is symptomatic and initially involves management in intensive care (oxygen therapy, hydroelectrolyte correction and antibiotic therapy if signs of infection). Then, in a second time, cutaneo-mucous care by the early excision of necrosis and by oily tulle dressings on the discoloured surfaces for epidermal regeneration usually in two or three weeks. Pigmentation disorders (hypopigmentation and/or hyperpigmentation) are almost constant [26]. However, the management of the ocular damage may need aggressive topical corticosteroids and lubricant eye drops without preservatives and antibiotics. In some cases, amniotic membrane graft may be necessary in the acute phase to prevent or reduce long-term eye sequelae. On the other hand, the use of high-dose systemic corticosteroids or intravenous immunoglobulins remains highly controversial or even discouraged due to the increased risk of kidney failure and mortality [23]. In the chronic phase, the use of systemic immunosuppressants may be proposed for the preoperative control of persistent inflammation. The treatment of sequelae is symptomatic, it is based on: the prevention of dry eye syndrome, reconstructive surgery of fibrosis and conjunctival adhesions, palpebral malpositions, as well as management of corneal opacities [22]. 
In addition, the results of our study suggest that there is a significant correlation between the severity of ocular involvement and the importance of the lifted cutaneous surface as well as with the overall severity of the disease. This is quite evident while seeing the association of severe stages according to the Foster classification to the most severe stages of SCORTEN predictive survival score. Several studies share our theory, including a Congolese study conducted by Bagamboula et al. [19] and a Chinese study published in 2017 by Lorraine et al. [26]. However, an American study by Hajirah et al. refutes this theory, but states that the degree of chronic ocular inflammation depends on the intensity of ocular involvement in the acute phase [27].

\section{CONCLUSION}

The syndromes of Lyell and Stevens Johnson are severe and life-threathning, hence the need for early and adequate multidisciplinary management with regular follow-up to avoid sequelae. There is a direct correlation between the severity of cutaneous and eye damage.

\section{Declaration of Interest} of interest.

The authors declare that they have no conflicts

\section{REFERENCES}

1. Sassolas B, Haddad C, Dunant A, ValleyrieAlanore L, Rou-jeau JC. Analysis over 10 years. Ann DermatolVenereol. 2011; 138:89-92.

2. Fu Y, Gregory DG, Sippel KC, Bouchard CS, Tseng SC. Theophthalmologist's role in the management of acute Stevens-Johnson syndrome and toxic epidermal necrolysis. Ocul Surf. 2010;8:193-203

3. Bastuji-Garin S, Fouchard N, Bertocchi M, Roujeau JC, RevuzJ, Wolkenstein P. SCORTEN: a severity-of-illness score for toxicepidermal necrolysis. J Invest Dermatol. 2000; 115:149-53.

4. Bastuji-Garin S, Rzany B, Stern RS, Shear NH, Naldi L, RoujeauJC. Clinical classification of cases of toxic epidermal necro-lysis, Stevens-Johnson syndrome, and erythema multiforme.Arch Dermatol. 1993; 129:92-6.

5. Foster CS. Cicatricial pemphigoid. Trans Am Ophthalmol Soc.1986;84:527-663.

6. Bégaud B, Evreux JC, Jouglard J, Lagier G. Imputabilité deseffets inattendus ou toxiques des médicaments. Actualisationde la méthode utilisée en France. Therapie. 1985;40:111-8.

7. Rzany B, Mockenhaupt M, Baur S, Schröder W, Stocker U, Mueller J. Epidemiology of erythema exsudativum multiformemajus. Stevens-Johnson syndrome, and toxic epidermal necrolysis in Germany (1990-1992): structure and results of a population-based registry. J Clin Epidemiol. 1996;49:769-73.
8. Roujeau JC, Stern RS. Severe adverse cutaneous reactions to drugs. N Engl J Med. 1994; 331:127285.

9. Valeyrie-Allanore L, Ingen-Housz-Oro $\mathrm{S}$, Chosidow O, Wol-kenstein P. French referral center management of Stevens-Johnson syndrome/toxic epidermal necrolysis. Dermatol Sin. 2013; 31:191-5.

10. Halebian PH. A burn center experience: Toxic epidermal necrolysis. Dermatology. 1993; 186:3237.

11. Mouafik SB, Hocar O, Akhdari N, Amal S, Belghmaidi S, Ennassiri W, Hajji I, Moutaouakil A. Manifestations ophtalmiques au décours des syndromes de Lyell et de Stevens-Johnson. InAnnales de Dermatologie et de Vénéréologie 2015 Jun 1 (Vol. 142, No. 6-7, pp. 393-398). Elsevier Masson.

12. Watanabe R, Watanabe H, Sotozono C, Kokaze A, Iijima M. Critical factors differentiating erythema multiforme majus fromStevens-Johnson syndrome (SJS)/toxic epidermal necrolysis(TEN). Eur J Dermatol. 2011;21:889-94

13. Kourouma S, Sangare A, Kaloga M, Kouassi I, Ecra E, Gbery I. Syndromes de Stevens-Johnson et de Lyell : étude de185 cas au CHU de Treichville (Abidjan, Côte d'Ivoire). MedSante Trop. 2014;24:94-8.

14. Moussala M., Behar Cohen P., Dighiero P., Renard G. Le syndrome de Lyell et ses manifestations ophtalmologiques en milieu camerounais. J. Français d'Ophtalmologie. 2002;23:229-237.

15. Couadau E., Carles M., Ichai C. Conférence d'actualisation. Paris: Elsevier. Troubles cutanés en réanimation. 2001; 551-570.

16. Dikhaye Siham. Syndrome de Lyell et syndrome de Stevens-Johnson: l'expérience du service de dermatologie du centre hospitalier universitaire Ibn-Sina, Rabat, Maroc. Thèse No ${ }^{\circ} .2005 ; 107$.

17. Chosidow O, Roujeau JC, Revuz J. Syndrome de Lyell (nécrose épidermique toxique) EMC. 1991;12450:A10-A10.

18. Ophtalek A, Brown K, Sens S, Halerz M, Supple K, Gameli RL. Long-term follow-up of patients treated for toxic epidermalnecrolysis. J Burn Care Res. 2006;27:26-33.

19. Bagamboula M, Gathse A, Lenga Loumingou I, Kaya Ganziami G. Les complications oculaires au cours des syndromes de Stevens-Johnson et de Lyell. J Fr Ophtalmol. 2007;30.

20. Atipo-Tsiba PW, Bayoune S, Messe Ambia R. Atteintes ocu-laires au cours des toxidermies médicamenteuses : à proposde 10 cas. Mali Med. 2011;26:65-8.

21. Fellahi A, Zouhair K, Amraoui B, Benchikhi H. Séquelles cuta-néomuqueuses et oculaires des syndromes de Stevens-Johnsonet de Lyell. Ann Dermatol Venereol. 2011;138:88-92. 
22. Gaudry J, Muraine M. Syndrome de StevensJohnson et Lyell.Reflexions Ophtalmologiques. 2007; 103:20-3.

23. Yip LW, Thong BY, Lim J, Tan AW, Wong HB, Handa S.Ocular manifestations and complications of Stevens-Johnsonsyndrome and toxic epidermal necrolysis: an Asian series. Allergy. 2007; 62:527-53.

24. Gueudry J, Roujeau JC, Binaghi M, Soubrane G, Muraine M.Risk factors for the development of ocular complications ofStevens-Johnson syndrome and toxic epidermal necrolysis. Arch Dermatol. 2009; 145:157-62.

25. Morales E, Perdue F, Verity SM, Arnoldo B, Bloumouist P. Oph-thalmic manifestations of Stevens-Johnson syndrome and toxicepidermal necrolysis and relation to SCORTEN. Am J Ophthal-mol. 2010; 150:505-10.

26. Bachot N, Roujeau JC. Differential diagnosis of severe cutaneous drug eruptions. Ann J Dermatol. 2003; 4:561-572.

27. Chow LLW, Ng, ALK, Chow SSW. A crosssectional comparative study on chronic ocular manifestations of Stevens-Johnson syndrome and toxic epidermal necrolysis in Chinese eyes: a 15year case series. Int Ophthalmol. 2018; 38: 11551160.

28. Ocular manifestations of Stevens-Johnson syndrome and their management Saeed, Hajirah N; Chodosh, James Current Opinion in Ophthalmology. 2016; 27(6):522-529. 